\title{
Special Issue 2016
}

This Special Issue of the Journal of Dynamics and Differential Equations, consisting of the combined September-December 2016 issues of the Journal, is dedicated to Professor John (Joseph Douglas) Mallet-Paret in celebration of his 60th birthday.

Professor Mallet-Paret is a leading expert in infinite dimensional dynamical systems especially those generated by functional differential equations. He has been serving on the editorial board of this journal since its founding.

With 28 invited articles including a biographical article written by Shui-Nee Chow, Roger Nussbaum, and Hans-Otto Walther, the Special Issue has been in preparation since 2013 in conjunction with a Workshop on Dynamics of Differential Equations held at Brown University in 2012 in Professor Mallet-Paret's honor.

We are very pleased that Aaron Hoffman, Roger Nussbaum, Hans-Otto Walther, and Jianhong Wu have joined with Yingfei Yi and George R. Sell as special editors for this Issue, and we sincerely thank them for their valuable assistance. Professor George R. Sell, co-editor in chief of the Journal, who sadly passed away on May 29, 2015, had been of great help in planning and editing this Special Issue.

Geneviève Raugel and Yingfei Yi

Co-Editors in Chief

Geneviève Raugel

CNRS, Laboratoire de Mathématiques, University Paris-Sud, Orsay, France

e-mail: genevieve.raugel@math.u-psud.fr

Yingfei Yi

University of Alberta, Edmonton, Canada

Georgia Institute of Technology, Atlanta, GA, USA

e-mail: yingfei@ualberta.ca; yi@math.gatech.edu 


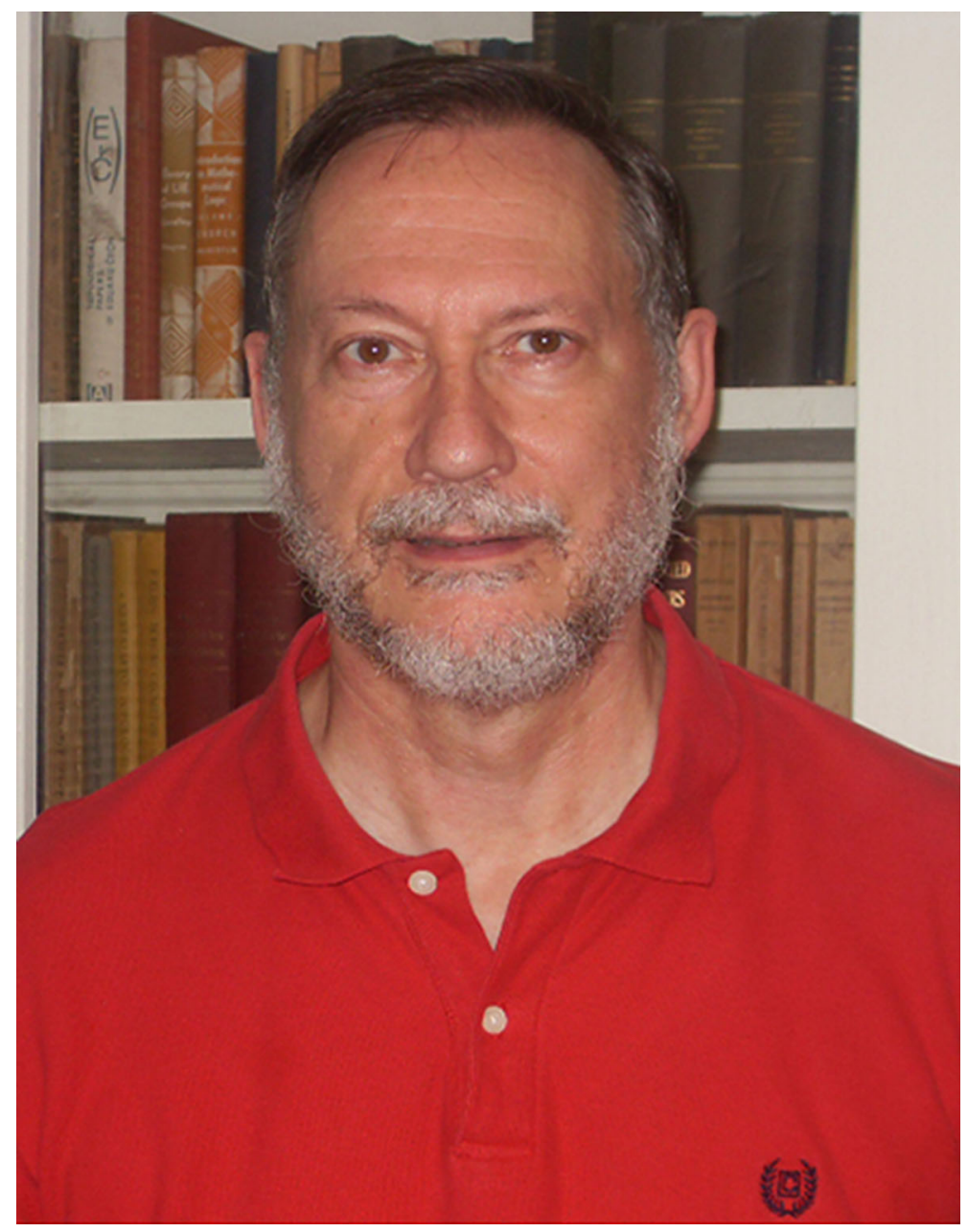

Professor John (Joseph Doulags) Mallet-Paret 